\title{
Gene activities and segmental patterning in Drosophila: analysis of odd-skipped and pair-rule double mutants
}

\author{
Douglas E. Coulter ${ }^{1}$ and Eric Wieschaus \\ Department of Biology, Princeton University, Princeton, New Jersey 08544 USA
}

\begin{abstract}
The odd-skipped (odd) gene is required to generate anterior regions of the odd-numbered segments in Drosophila; homozygous embryos show pattern deletions that are always less than a segment in width and are associated with mirror-image duplications of adjacent regions. To define further the role of odd and determine how it interacts with other segmentation genes, we have described the effects of combining odd with mutations at other pair-rule loci. We have observed phenotypic suppression in double-mutant combinations with evenskipped (eve), paired, sloppy paired, and engrailed (en). In the most thoroughly characterized combination (odd eve), both naked cuticle and specific denticle rows are restored that would normally have been deleted by one of the two mutants alone. In the odd en double mutant, we observe nearly complete suppression of the odd phenotype, such that the mirror image duplications are eliminated and the odd-numbered denticle bands are restored. We conclude that the requirements of pattern elements for specific gene activities are not absolute, and propose mechanisms by which these genes interact to specify cell fates.
\end{abstract}

[Key Words: Pair-rule mutants; Drosophila segmentation]

Received July 11, 1988; revised version accepted October 3, 1988.

The pair-rule genes of Drosophila are required during embryogenesis for the establishment of homologous elements in every other segment (Nüsslein-Volhard and Wieschaus 1980; Gergen et al. 1986). To date, mutations in eight pair-rule genes have been identified, all of which produce pattern deletions spaced at double-segment intervals. The region of the pattern deleted in mutant embryos is locus specific, indicating that each pair-rule activity is required to specify cell fates in unique domains of the segmental pattern. Both the autonomy observed in genetic mosaics (Gergen and Wieschaus 1985) and the transient double-segment 'stripes' of expression at the blastoderm stage (reviewed in O'Farrell and Scott 1986; Akam 1987) suggest that these genes act primarily in subsets of cells that correspond to the regions deleted in the mutant patterns. While the pattern of pair-rule expression does not persist beyond early embryogenesis, at least one important function of pair-rule genes is the proper activation of more stably expressed genes (e.g., engrailed, Kornberg et al. 1985; DiNardo and O'Farrell 1987; and wingless, Ingham et al. 1988). These downstream genes appear to maintain the segmental pattern at later stages.

'Current address: Department of Biochemistry, St. Louis University Medical Center, St. Louis, Missouri 63104 USA.
In the experiments reported here, we have focused on one pair-rule gene, odd-skipped (odd). We show that pattern deletions in mutant embryos are restricted to discrete domains in the overall pattern and that their size varies with the strength of the odd allele. This variability allows us to define a relationship between the apparent level of odd activity and specific pattern elements. However, because any given element in the segmental pattern is affected by more than one pair-rule gene, an understanding on the relationship between odd and the final pattern must take into account its interactions with the other pair-rule genes. To analyze these interactions, we made double mutants of odd with each of the other pair-rule genes. If the pattern deletions in single mutants reflect absolute requirements for the corresponding gene activity, the effects of combining two mutations should be additive, with the double-mutant phenotype reflecting the sum of the two pattern deletions. If, on the other hand, final pattern depends on the ratio of segmentation gene products, or on regulatory interactions between pair-rule genes, the loss of pattern elements associated with one locus might be alleviated by changes in the activity of the other. Based on the results of such double-mutant combinations, we present models for the role of odd in the global segmentation process and show how its interactions with other pairrule genes might specify cell fates. 


\section{Results}

\section{Odd cuticle phenotype}

Homozygous odd mutants develop to the end of embryogenesis, secrete an abnormal cuticle, and fail to hatch. The observed defects are spaced at double-segment intervals and can be described as deletions of particular pattern elements. Deletions of prominent features in the odd-numbered abdominal segments and in the mesothorax (T2, A1, A3, A5, and A7) are detailed below (Fig. 1a). Other deleted or defective structures include the anal tuft and labial derivatives including the hypophysis, the anterior regions of the $\mathrm{H}$ piece, and the dorsal anchors of the mouth hooks. In addition, the dorsal pouch is shortened, resulting in the close juxtaposition of the dorsal arm of the cephalopharyngeal skeleton and the medial tooth of the labrum; the latter is frequently misshapen, as well (see Jürgens et al. 1986).

Mutations in odd define an allelic series, with phenotypes classified as strong (4 alleles), moderate (2 alleles), or weak ( 1 allele) based upon the frequency and extent of pattern deletions (see also descriptions of hairy, Ingham et al. 1985; runt, Gergen and Wieschaus 1986). The cuticle pattern of a late embryo homozygous for a weak odd mutation is shown in Figure 1, $\mathrm{c}$ and $\mathrm{f}$. The observed deletions affect only the 'odd-numbered' segments, but the expressivity of the phenotype is low, as defects within a given segment are often subtle or undetectable. The weak mutation rarely deletes a complete denticle belt, but instead shows a marked tendency to affect the posterior denticle rows while leaving the anterior region of the belt intact. The deletion of posterior structures is associated with a duplication in reversed polarity of the remaining anterior rows. For example, the third abdominal denticle belt shown in Figure lf exhibits one normal and one defective half. On the defective side, the orientation and polarity of the anterior denticle rows is normal, but the posterior rows represent a rough mirror image of the anterior pattern. Thus, the phenotype represents the deletion of those pattern elements that normally lie posterior to the mirror plane, coupled with the duplication of the elements anterior to this plane.

In stronger phenotypes, both the frequency and severity of observed defects increases, reflecting an expanded deletion frame. In the strongest phenotype (Fig. le,h), the odd-numbered denticle belts are deleted altogether, or more often are reduced to just one or two duplicated anterior rows. The moderate phenotype (Fig. $1 \mathrm{~d}, \mathrm{~g}$ ), has an average deletion interval between those exhibited by severe and weak alleles. Regardless of the extent of the deletions, the remaining anterior rows exhibit pattern duplications with reversed polarity. In all three phenotypes, the even-numbered belts remain unaffected.

Two observations suggest a graded requirement for odd activity among the affected pattern elements. First, within each odd-numbered segment, the deletion frame is centered over the naked cuticle, at a position anterior to the middle of the segment but posterior to the denticle belt. Depending upon the severity of the odd mutation, pattern deletions extend from this position into the adjacent pattern elements and range in size from onehalf to approximately three-quarters of a segmental repeat. In addition to this differential sensitivity within segments, the phenotype also reveals a skewed 'regional specificity,' such that within a given embryo the frequency of defects varies among the odd-numbered segments. Most odd alleles exhibit the same regional specificity, with the probability and/or severity of deletions decreasing in the following order: labral, labial $>$ tuft,

Figure 1. The odd phenotype. (a) A schematic representation of the wild-type larval cuticle (top), with regions deleted in odd mutants delineated by boxes, and the resulting cuticle phenotype (bottom). The deletion intervals shown approximate an average for the strong phenotype, except that those indicated for the third, fifth, and seventh abdominal segments (A3, A5, A7) are somewhat reduced to illustrate better the polarity reversals (arrows) in the mutant phenotype; note that the boundaries of the deletions (which correspond to mirror planes in the mutant phenotype) do not necessarily correspond to segment boundaries. $(b)$ The wild-type cuticle pattern, showing a dark-field photomicrograph of a first instar larva. The anterior of each thoracic and abdominal segment is marked by a belt of ventral denticles arranged in a characteristic pattern of rows. $(c)$ A weak phenotype of a late embryo homozygous for odd IIIC. Note the defects in the posterior rows of the third (A3) and fifth (A5) abdominal denticle belts, the deletion of the right half of the Al belt, and the normal pattern in A7. (d) A moderate phenotype generated by an oddIIIC/oddIII heterozygote; embryos homozygous for $o d d^{9 P}$ exhibit a similar range of phenotypes. In the embryo shown, all odd-numbered segments exhibit defects, ranging from the complete absence of the Al denticle belt to deletion of only the posterior rows in half of the A5 belt. (e) An embryo homozygous for a strong allele, odd ${ }^{I I D}$. All of the odd-numbered segments exhibit strong pattern deletions; denticles are completely absent from $\mathrm{A} 1$ and $\mathrm{A} 7$, while only the anterior rows remain in $\mathrm{A} 3$ and $\mathrm{A} 5 .(f-h)$ Phase-contrast photomicrographs of the ventral A2-A3 region of mutant embryos homozygous for weak (odd $\left.{ }^{I I C}\right)$, moderate $\left(o d d^{P P}\right)$, and strong (odd $\left.{ }^{7 L}\right)$ alleles. The polarity of the pattern is indicated by arrows, which point posteriorly in normal and anteriorly in reversed regions; note that the pattern and polarity of the right half of the A3 belt in $f$ is normal. The polarity within each belt is indicated by the orientation of individual denticles and by variations in denticle size and shape which characterize each row. The approximate positions of reversals (mirror planes) in ventral regions of naked cuticle are inferred primarily from the analysis of the finely graded pattern of hairs on the dorsal surface, where obvious polarity reversals mark both boundaries of a given deletion; these dorsal deletions consistently affect less than a whole segment, leaving the posterior elements of the odd segments intact. Also, the ventral Keilin's organ derived from the second thoracic segment is often present and sometimes duplicated in strong mutants, consistent with a boundary in this region. Abbreviations (see Jürgens et al. 1986; Jürgens 1987 for complete descriptions): $M T$, median tooth; CPS, cephalopharyngeal skeleton; $M H$, mouth hooks; $L B S$, labial sense organ; $H$, H-piece; VP, ventral pit (sense organ); KO, Keilin's organ; $A P$, anal pad; $T F T$, anal tuft; $F Z$, filzkörper; mt, metathorax. 
$\mathrm{T} 2>\mathrm{A} 1>\mathrm{A} 7>=\mathrm{A} 3>\mathrm{A} 5$. Thus, it appears that different levels of odd activity are required in different regions, with, for example, a greater requirement in the first abdominal segment than in the fifth.

\section{Cuticle phenotypes of even-skipped embryos}

To evaluate better the interactions between the odd and even-skipped (eve) loci, we first examined the cuticle phenotype of three eve alleles. These include one that yields a weak phenotype and a deficiency that produces the null phenotype. The third, a temperature-sensitive allele, exhibits a broad range of phenotypes, overlapping the weak phenotype at $18-20^{\circ} \mathrm{C}$ and approaching the amorphic phenotype at $31-33^{\circ} \mathrm{C}$.

In the weakest eve phenotype, mutant embryos show a variable tendency to delete even-numbered denticle belts. In contrast to the odd phenotype, the deletions are centered over (rather than posterior to) the denticle belts; when deletions occur they affect all the denticle rows in the belt (see A2 and A6 in 'eve $e^{w '}$ phenotype, Fig. 2 ), in contrast to the partial deletions of posterior rows characteristic of the odd phenotype. As the eve phenotype becomes progressively more severe (e.g., using the $t s$ allele at higher temperatures), the probability of deleting the even-numbered denticle belts increases. These probabilities are graded, reflecting different regional specificities along the anterior-posterior axis of the em-
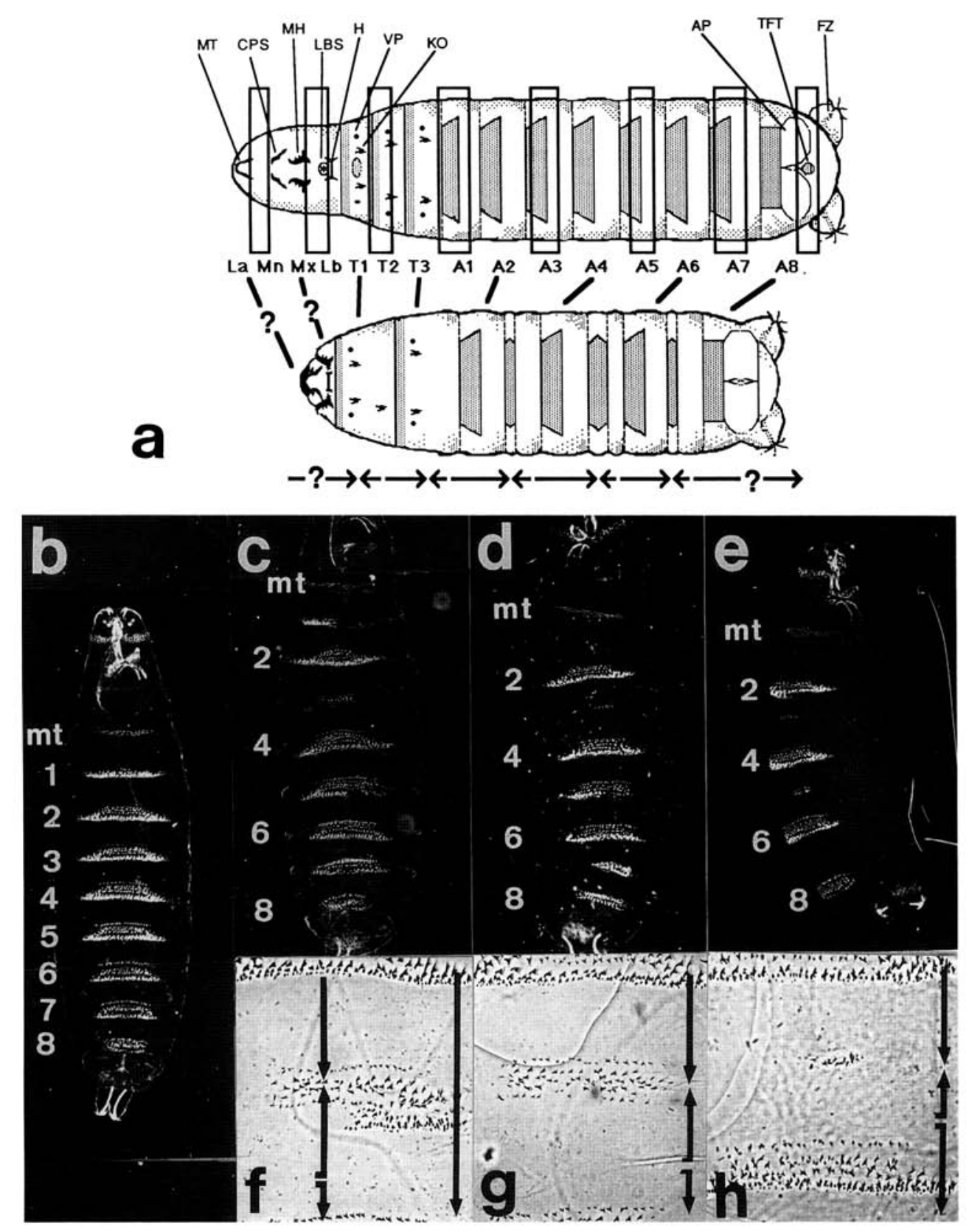

Figure 1. (See previous page for legend.) 


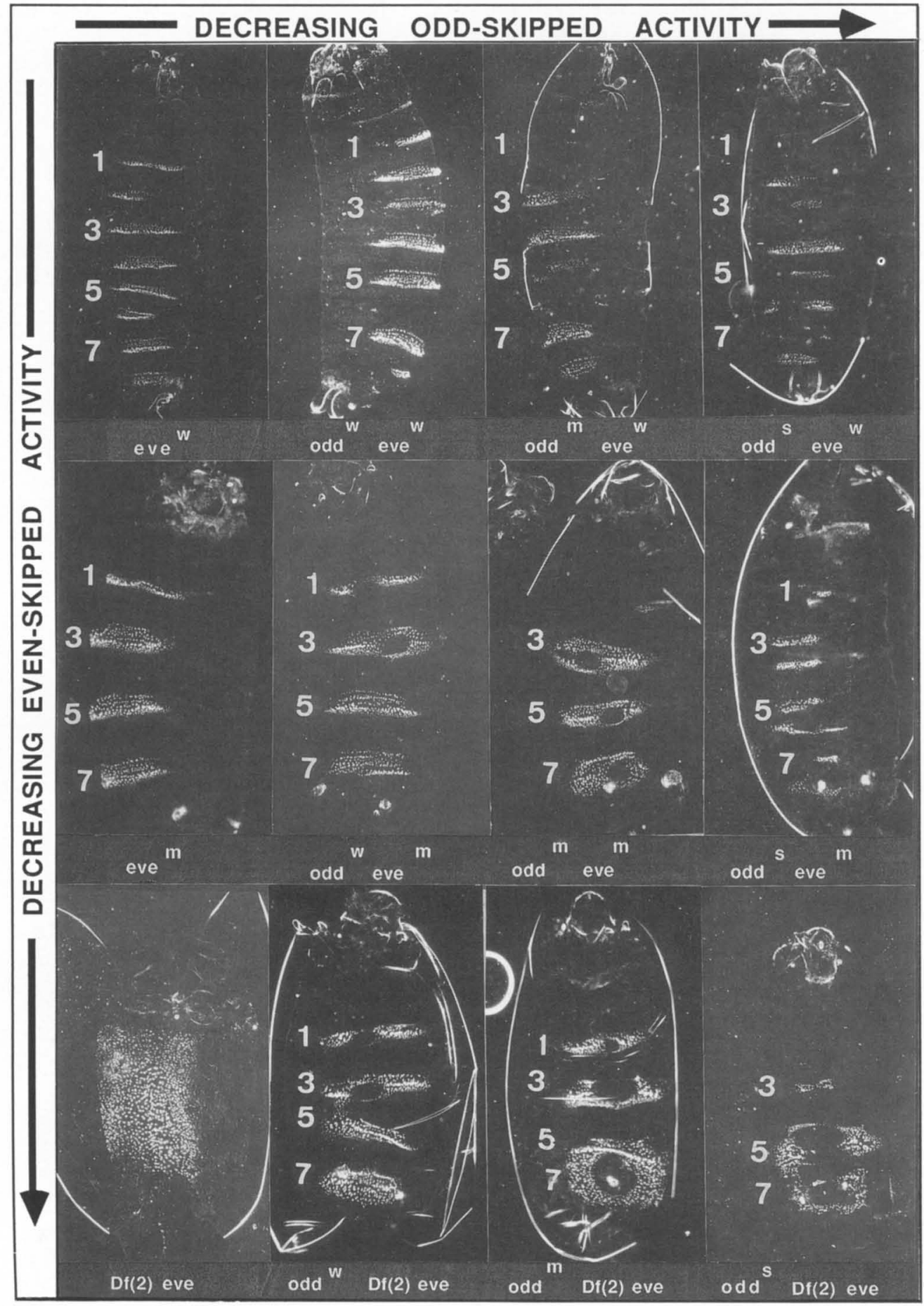

Figure 2. Phenotypes of $e v e$ and odd eve double mutants. Cuticle phenotypes of weak $\left(e v e^{w}\right)$, moderate or pair-rule $\left(e v e^{m}\right)$, and amorphic $[D f(2) e v e]$ eve embryos homozygous for $e v e^{I D}\left(18^{\circ} \mathrm{C}\right)$, eve $e^{I D}\left(29^{\circ} \mathrm{C}\right)$, and $D f(2) e v e^{1.27}$, respectively, are shown in the first column. Successive columns show these same alleles (or growth temperatures) in combination with odd ${ }^{\text {IIIC }}$ (homozygous) (=oddw), odd ${ }^{I I C} /$ odd ${ }^{I I D}$ (transheterozygous) (=odd ${ }^{m}$ ), or odd $d^{I I D}$ (homozygous) (=odds), except that the odd $/$ evew combination had $e v e^{I I R}$ and was raised at $25^{\circ} \mathrm{C}$ (in place of $e v e^{I D}$ at $18^{\circ} \mathrm{C}$ ) and the odd $e v e^{m}$ animal shown was heterozygous for odd $d^{I I C} /$ odd $d^{7 L}$. The most obvious difference between the pair-rule (eve $e^{m}$, middle row) and amorphic (bottom row) eve combinations is the tendency for adjacent compound belts to fuse laterally in the amorphic double mutants due to an incomplete restoration of naked cuticle. This tendency, which is most pronounced in more posterior regions and with the stronger odd alleles, should be contrasted with the alternately phased fusions (described in the text and illustrated in Fig. 3) which result as odd activity is increased. These latter fusions involve the elimination of regions with reversed polarity, while the partial loss of naked cuticle noted here results when postions of the pattern with normal polarity are eliminated. 
bryo $(\mathrm{A} 6>\mathrm{A} 2>\mathrm{A} 4>\mathrm{A} 8)$; thus, at $25^{\circ} \mathrm{C}$ the temperaturesensitive embryos commonly lack belts $\mathrm{A} 2$ and $\mathrm{A} 6$, but frequently retain all or part of A8 and sometimes A4. At $29^{\circ} \mathrm{C}$, the loss of even-numbered belts becomes complete, yielding the eve 'pair-rule' phenotype $\left(e v e^{m}\right.$ in Fig. 2 ). Here the regularly spaced odd-numbered denticle belts are essentially normal (although somewhat broadened) and fully separated by naked cuticle.

In embryos homozygous for a chromosomal deficiency of the eve locus (or for the $t s$ allele at $31^{\circ} \mathrm{C}$ ), the characteristic pattern of odd-numbered belts separated by naked cuticle is not observed; rather, the ventral abdominal region of the epidermis secretes a continuous mass or 'lawn' of denticles with no intervening naked cuticle (Nüsslein-Volhard et al. 1985; MacDonald et al. 1986, see also Fig. 2). Studies with the temperature-sensitive allele indicate that the lawn derives from the 'fusion' of odd-numbered denticle belts, due to the progressive loss of intervening naked cuticle as eve activity is further reduced from the $29^{\circ} \mathrm{C}$ level. Therefore, we believe that this amorphic phenotype reflects an extremely broad deletion frame that is still fundamentally 'pair-rule' in nature, with all positional values deleted except those corresponding to a few rows of odd-numbered denticle belts.

Several differences between the odd and eve phenotypes indicate that the two genes do not play complementary roles in specifying the pattern of segments. While defects in the strongest odd mutants affect less than a single segment, mutations in eve readily delete more than a segmental repeat, with the complete loss of function resulting in the apparently asegmental lawn of odd numbered denticles. In addition, the two pair-rule phenotypes do not affect complementary regions of alternate segments (see Fig. 4 and 5). Deletions in odd embryos are centered in the naked cuticle of the odd-numbered segments, and are largely limited to these segments. In contrast, the eve deletions are centered over the even-numbered denticle belts and readily extend into the posterior regions of the anteriorly adjacent oddnumbered segments, approximating a parasegmental rather than a segmental phasing (Martinez-Arias and Lawrence 1985). Finally, the two phenotypes can be distinguished by the presence or absence of polarity reversals, with the eve deletions resulting in the juxtaposition or 'fusion' of unaffected elements that retain normal polarity, while the odd deletions are coupled consistently with pattern duplications with reversed polarity.

Odd, eve double mutants

To examine the relationship between the odd and eve genes, we prepared all nine possible double mutants using the strong, moderate, and weak alleles described above for the two loci. When a weak mutation in eve is combined with either a weak, moderate, or strong odd allele (Fig. 2, top row), the resulting phenotype is essentially additive, with the effects on the even- and oddnumbered denticle belts reflecting independent effects of the two loci in different regions of the embryo (see top row of Fig. 4). Further reductions in eve activity resulted in unexpected phenotypes that deviate from additivity. In its most regular form (produced by combinations with the temperature-sensitive eve allele at $29^{\circ} \mathrm{C}$, middle row of Fig. 2), the double mutant exhibits seven or eight partial denticle belts. With stronger odd alleles $\left(o d d^{s}, e v e^{m}\right.$ in Fig. 2), these partial belts tend to be fully separated by naked cuticle, and each reveals an apparent polarity reversal (Fig. 3c). The odd-numbered belts show an expected pattern similar to that in odd mutants, with the posterior half of each belt representing a mirror image duplication of normal anterior rows. In contrast, the even-numbered belts represent a novel feature of the

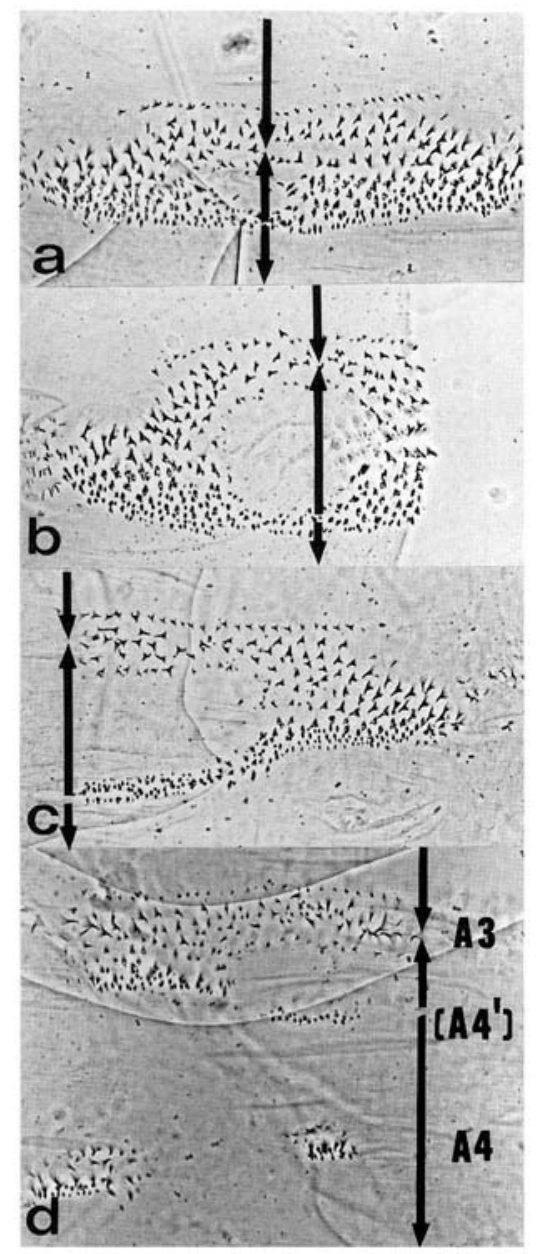

Figure 3. Phase-contrast micrographs of selected odd eve double mutants. $(a-c)$ The ventral A3/A4 region of an oddw/ $e v e^{m}, o d d^{m} e v e^{m}$, and odd $e v e^{m}$ embryo, respectively; for genotypes, see Fig. 2. Note that as odd activity is decreased, the anterior and posterior rows become separated by regions of reversed polarity (arrows) as regions with normal polarity are diminished. Assuming that the phenotype in $a$ approximates that of paired mutants (see Figs. 5 and 6) and represents the deletion of a single segmental repeat, this deletion interval appears to expand as odd activity is further reduced in $b$ and $c$, with the intercalation of a reversed polarity duplication when more then a single segment is deleted. (d) The ventral A3/A4 region of an odd $d^{I I D} e v e^{I D}$ homozygote raised at $25^{\circ} \mathrm{C}$. Note that remnants of the original A4 denticle belt are occasionally present at this temperature; these were never observed with the same genotype raised at $29^{\circ} \mathrm{C}$ (as in $\mathrm{c}$ ). 
odd eve interaction and show the reverse: Normal posterior rows are duplicated anteriorly (see Figs. 3 and 4). Although these are situated between the remaining anterior rows of the odd-numbered belts, it is unlikely that these denticles represent the restoration of a portion of the normal even-numbered belts. This is suggested by the 'transitional' phenotype of a similar double-mutant combination raised at a lower temperature (Fig. 3d). In this case, we frequently observed these novel, posterior rows in the region of naked cuticle anterior to a remnant of the even-numbered belt. Therefore, it appears that these even-numbered partial belts are derived ectopically from a region of naked cuticle within the odd-numbered segments.

In the odd ${ }^{s}$ ever phenotype (Figs. 2 and $3 \mathrm{c}$ ), the alternating pattern of polarity reversals within each partial denticle belt suggests that a continuous region of reversed polarity (including the reversed portions of each belt and the intervening naked cuticle, see Fig. 4) exists between each pair of belts. The presence of these polarity reversals depends upon the severity of the odd mutation: When odd activity is increased by using weaker alleles (holding the state of eve activity constant), the reversed regions are diminished and more elements are restored to both the anterior and posterior denticle rows. This results in the fusion of the anterior and posterior denticle rows into a single belt with normal polarity, a process that first occurs laterally with intermediate levels of odd activity (Fig. 2, odd ${ }^{\mathrm{m}} e \mathrm{ve}^{\mathrm{m}}$; Fig. $3 \mathrm{~b})$, and becomes essentially complete with the weakest odd mutation (Fig. 2, odd ${ }^{w} e v e^{m}$; Fig. 3a). Thus, the eight partial abdominal belts observed in double mutants with strong odd alleles give rise to four 'fused' belts as odd activity is increased and the intervening pattern duplications are eliminated.

The most obvious feature that results when a deficiency for the eve locus is combined with mutations in odd is the presence of naked cuticle separating denticle belts in the abdominal region (Fig. 2, bottom row). Thus, the reduction or elimination of odd activity tends to restore regions of naked cuticle and thereby transform the asegmental lawn of denticles in the amorphic eve phenotype into a periodic pattern. Although they are somewhat more variable, the resulting phenotypes can be interpreted using the same paradigm described for the analogous $' 29^{\circ} \mathrm{C}$ ' phenotypes described above (i.e., each of the denticle belts shows a mirror-image polarity reversal and the entire region between the odd and even belts shows inverted polarity, Fig. 4). The major difference between the two phenotypes is a tendency for the belts in the odd Df(2)eve embryos to fuse laterally.

\section{Other double mutants}

In addition to the detailed analysis of odd eve doublemutant combinations, we have examined the phenotypes of odd in combination with mutations in all other available pair-rule loci, and in many cases, with multiple alleles of those loci. The pattern deletions associated with the various single mutants are represented schematically in Figure 5; Figure 6 shows the ventral ab- dominal cuticle derived from each mutant and, where possible, the corresponding double-mutant phenotype in combination with a strong odd allele.

In combinations with four of the pair-rule mutants tested, the phenotype of the odd double mutant is essentially additive. In the two cases where the pattern deletion associated with the other pair-rule mutant was large enough to include totally that of odd (i.e., runt and fushi tarazul, this additivity meant that the double mutants could not be distinguished from the phenotype of the tested pair-rule mutant alone. In the other two cases (hairy and odd-paired), the double mutants showed a new, more severely deleted pattern (Fig. 6) in which the pattern elements eliminated by either mutant alone were eliminated from the double-mutant pattern as well.

The remaining three mutants tested (paired, sloppypaired, and engrailed) tended to suppress the odd phenotype in double-mutant embryos, in that the size of the odd deletion is reduced and the mirror image eliminated. The suppression is most striking in the engrailed (en) combination (Fig. 6), because en mutations primarily affect the posterior naked cuticular regions of each segment, i.e., a region outside the odd domain. In odd en double mutants, the odd-numbered denticle belts are restored and all vestiges of a mirror-image duplication are suppressed. The animals still show the fused denticle belts characteristic of en pattern deletions, but these occur less frequently and the overall appearance of the double mutant is more similar to that produced by hypomorphic alleles at $e n$. In double mutants combining odd with paired or sloppy-paired, a similar suppression of the mirror image duplication is observed, but the restoration of elements deleted by odd is less complete. However, in the odd paired double mutant, certain elements deleted in both single mutants are restored, indicating mutual suppression (Fig. 6).

\section{Discussion}

The role of the odd gene

Two features of the odd phenotype indicate its unique position among the pair-rule genes identified to date. The first is the relatively small size of the odd pattern deletions and the second is the consistent pattern of reversed polarity duplications observed in odd mutants. While mutations in most other pair-rule loci can cause the deletions of at least half or more of each double-segment repeat, the pattern deletions associated with odd always involve less than a whole segment. The coupling of these deletions with pattern duplications, in which some of the remaining elements of the pattern are reiterated in reversed polarity, readily contrasts with most other pair-rule phenotypes (with the exception of runt; see below), in which pattern elements adjacent to the deletion simply 'fuse'.'

The extent of the pattern duplication in an odd mu-

${ }^{1}$ Certain other pair-rule mutants do occasionally exhibit small regions of reversed or indeterminate polarity where disparate elements are juxtaposed; however, these are much more limited than the odd duplications and appear to represent only a very localized and incomplete 'smoothing' of the pattern. 

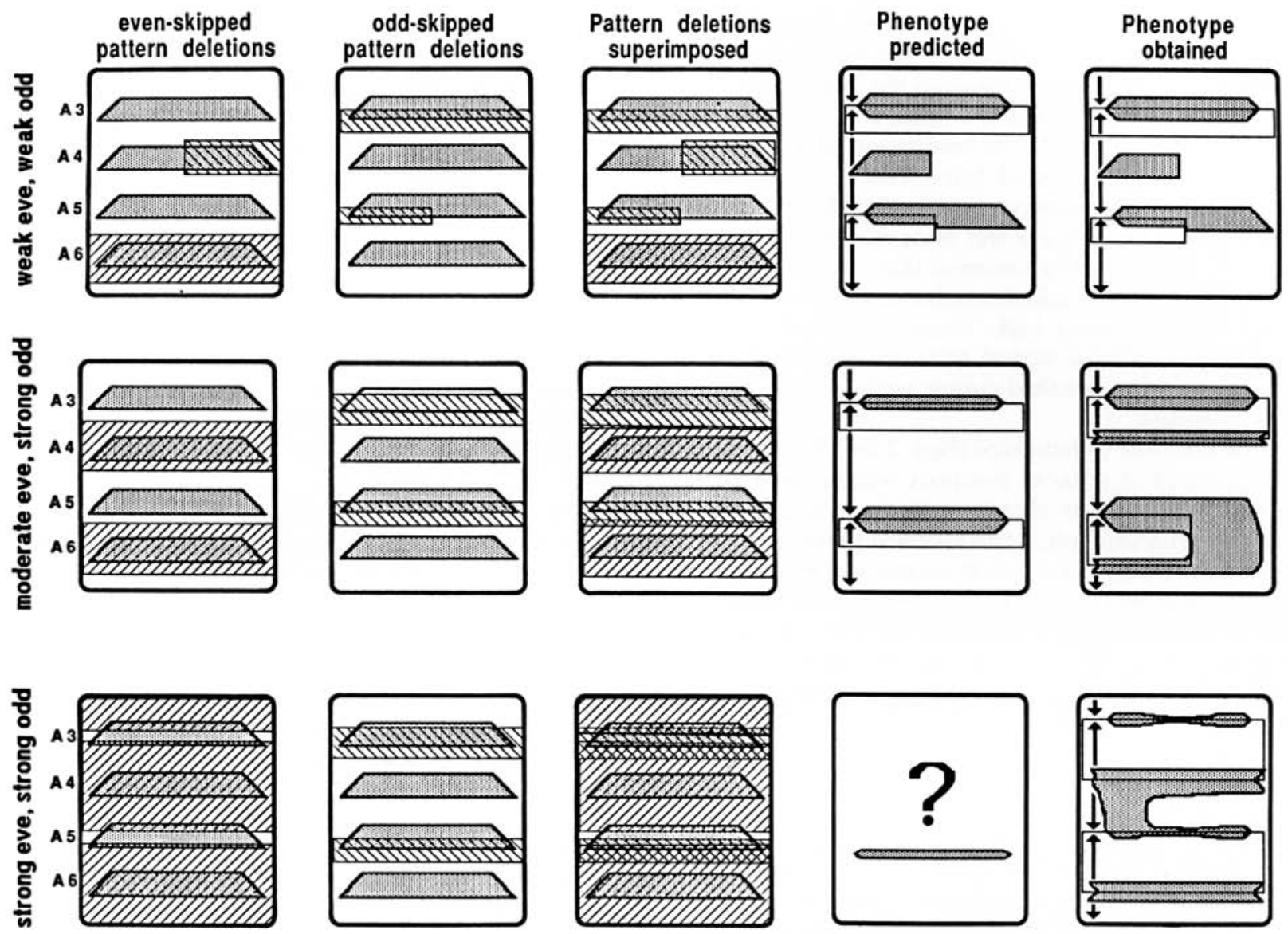

Figure 4. A schematic interpretation of the odd eve phenotype. Three different idealized double-mutant combinations are shown to illustrate the effects of varying the activity at the two loci. Shaded areas approximate the pattern deletions associated with each locus; these are combined to predict additive phenotypes, and these in turn are contrasted with representations of the denticle patterns observed in the actual phenotypes.

tant is related inversely to the size of the pattern deletion, such that both margins of the deletion behave as mirror planes (see Fig. 1). Furthermore, we have not observed the deletion of less than half a segment in even the weakest odd mutant, either in association with a pattern duplication or a simple fusion. Instead, the odd phenotype reveals a transitional jump from an essentially normal pattern to variably sized but coupled deletions and pattern duplications (e.g., Fig. 1f). Therefore, this phenotype formally mimics pattern regeneration mechanisms that have been described for circular or periodic fields (French et al. 1976), whereby 'shortest route' intercalation regenerates either a normal pattern or a reversed polarity duplication, depending on whether less or more than half of the positional values in a developmental field have been excised.

The only other pair-rule phenotype that exhibits a consistent pattern of polarity reversals, runt, differs from odd in two obvious respects. First, the runt duplications only occur upon the deletion of at least a full segmental repeat, suggesting by analogy to pattern regeneration mechanisms (see above) that the relevant developmental field for runt is a double-segment interval, whereas the odd duplications respond in a single segment mode, despite the obvious pair-rule (double segment) pattern of the odd deletions. Second, weaker runt alleles generate deletions of less than a segment that result in simple fusions, while no equivalent deletion of less than 'half of the field' (i.e., a half-segment) is exhibited by odd. These differences might be attributed to a greater complexity of the runt locus, with runt product being required at different places at different times. On the other hand, the runt and odd polarity reversals do share at least one common feature: Both result primarily from the respecification of cells that are affected by the mutation, and not as the product of cell death and 'shortest route' intercalary regeneration. In the case of runt this was inferred from the behavior of genetic mosaics (Gergen and Wieschaus 1985). With odd, this conclusion is based upon the pattern of en expression in odd mutants (DiNardo and O'Farrell 1987), and reinforced by the analysis of odd en double mutants reported here.

Using antibodies directed against the en protein, $\mathrm{Di}$ Nardo and O'Farrell (1987) observed that a strong muta- 


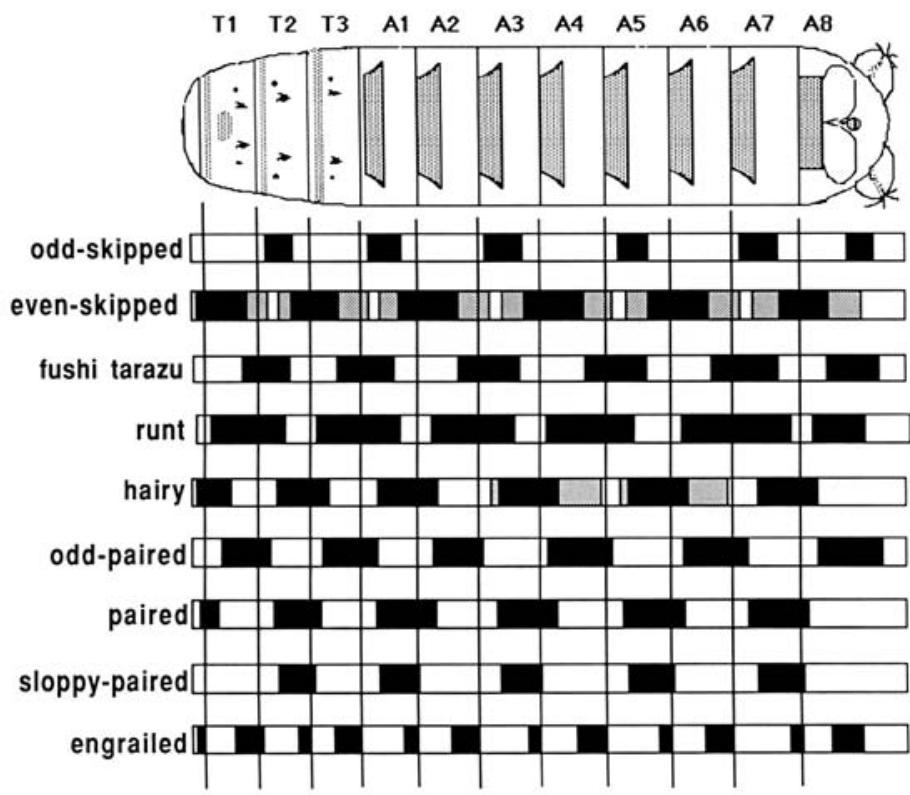

Figure 5. A schematic representation of pair-rule phenotypes. The pattern deletions associated with each phenotype are indicated by darkened bars. In general, these represent the phenotypes of the (strong) alleles used in this study. The shaded bars are shown to represent the more extreme pattern deletions in the strongest eve and hairy phenotypes.

Figure 6. (See p. 1820.) Pair-rule mutant phenotypes and corresponding double mutants with odd. Double mutants were generated and analyzed as described in Materials and methods. $f t z^{7 B}$ and runt $t^{I I I}$ : Strong mutations in both $f t z$ and runt completely eliminate the odd-numbered belts and surrounding regions of naked cuticle, affecting all of the elements deleted in the odd phenotype, with the possible exception of the more posterior regions of naked cuticle affected by odd. Considering this large overlap, additive combinations of the phenotypes in double mutants should closely resemble $f t z$ or runt alone. In fact, odd;runt and odd;ftz double mutants could not be distinguished unambiguously from single-mutant runt or ftz embryos, respectively. hairy ${ }^{I L}$ : The hairy mutation normally deletes the even-numbered denticle belts and the preceding naked cuticle. It also tends to delete all naked cuticle in the posterior abdomen. odd ${ }^{I I D}$; hairy ${ }^{I L}$ : The double mutant typically exhibits three or four partial denticle belts that appear to represent the anterior remnants of the odd-numbered segments expected for the odd mutation. The A1 and A3 denticle rows show obvious polarity reversals and are separated by regions of naked cuticle, whereas A5 and A7 are usually fused and the denticles reveal an indeterminate polarity. The double-mutant phenotype appears to be additive. odd ${ }^{9 \mathrm{C}}$ : The pattern in opa embryos consists of fused composite denticle belts, with a phasing opposite that of the paired phenotype. Anterior rows consist of 'even-derived' denticles, while posterior rows are odd; similar to weaker paired alleles, an incomplete fusion of the segments was occasionally observed with the opa mutant used here. In an additive double mutant, the elimination of odd activity should result in the deletion of the posterior (odd-derived) denticle rows from these composite belts. The remaining elements would include the anterior portions of the even-derived belts separated by naked cuticle, with an occasional remnant of the odd belts. odd ${ }^{I I D}$ odd-paired ${ }^{9 C}$ : The double-mutant phenotype is quite variable, but is generally consistent with additivity-three or four primary belts that are followed occasionally by patches of denticles. paired ${ }^{I N}$ (homozygote raised at $29^{\circ} \mathrm{C}$ ): The strong paired phenotype represents the deletion of all naked cuticle from the odd-numbered segments as well as the adjacent posterior denticle rows from the odd belts and the anterior rows from the even belts. In the abdominal cuticle, the result is four composite denticle belts comprised of anterior rows derived from odd-numbered segments and posterior rows from even-numbered segments. The paired deletions therefore represent a whole segmental repeat that overlaps all but the most anterior elements deleted by odd mutations. An additive sum of the two phenotypes should therefore result in a slight anterior extension of the paired domain. odd ${ }^{I I D}$ paired $d^{I I N}$ (homozygote at $29^{\circ} \mathrm{C}$ ): The deletion interval in the double mutant tends to be much more limited than expected from strict additivity, with the frequent restoration of both odd- and even-derived denticle rows and intervening regions of naked cuticle. Because these elements are preferentially restored to the region surrounding the ventral midline, pairs of belts commonly surround a 'bubble' of naked cuticle. The denticles adjacent to these bubbles usually exhibit normal polarity. We conclude that the two mutations tend to be mutually suppressive, resulting in a double mutant that resembles a weaker paired phenotype. sloppy-paired ${ }^{7 L}$ : Mutations in sloppy-paired reveal a domain similar to that of paired, but the lower and more variable expressivity of the sloppy phenotype results in a high frequency of 'unpaired' denticle belts separated by naked cuticle. odd ${ }^{7 L}$ sloppy-paired ${ }^{7 L}$ : In combination with a strong mutation in odd-skipped, the belts are more consistently 'paired', indicating a domain for the double mutant that resembles a strong paired phenotype and is roughly additive. However, the absence of polarity reversals indicates that sloppy-paired suppresses this aspect of the odd phenotype, and the posterior regions of naked cuticle appear to be deleted more frequently than in slp single mutants, suggesting a quantitative enhancement by odd. en ${ }^{I K}$ : Mutations in the en gene eliminate the posterior regions of naked cuticle from each segment, and variably delete the more anterior elements, resulting in a tendency for the denticle belts to fuse partially. This tendency is more pronounced in the even-numbered segments, indicating a pair-rule alternation in the severity of defects. odd $d^{I I D} e n^{I K}$ : The double mutant reveals a phenotype that is very similar to a weaker en phenotype. The consistent presence of the odd-numbered denticle belts, which show none of the defects and corresponding polarity reversals observed in odd mutants, indicates that en largely supresses the odd phenotype. 
tion in odd altered the early en pattern in a manner consistent with the cuticle phenotype described here. In odd mutant embryos, en expression still occurred in its normal domains, the posterior compartments of each segment. These regions are largely unaffected in the odd cuticle phenotype. Instead, every other segment expressed an ectopic, duplicated en domain, which apparently marked the cells that ultimately give rise to the duplicated posterior compartment in the odd cuticle phenotype. This suggests that at least a portion of the duplication results from the respecification of blastoderm cells prior to any cell death or division. Based on the phenotypic suppression we have observed in the odd en double mutant, it is clear that the en product not only serves to mark this respecification, but also plays a primary role in mediating the odd phenotype, presumably as a direct result of the ectopic expression. Thus, eliminating en function from this region in the double mutant results in the suppression of the reversed polarity duplications and, even more surprisingly, in the restoration of the deleted odd-numbered denticle rows. We conclude that at least one critical function of the odd gene product at the blastoderm stage is somehow to repress this ectopic expression of en (see also below).

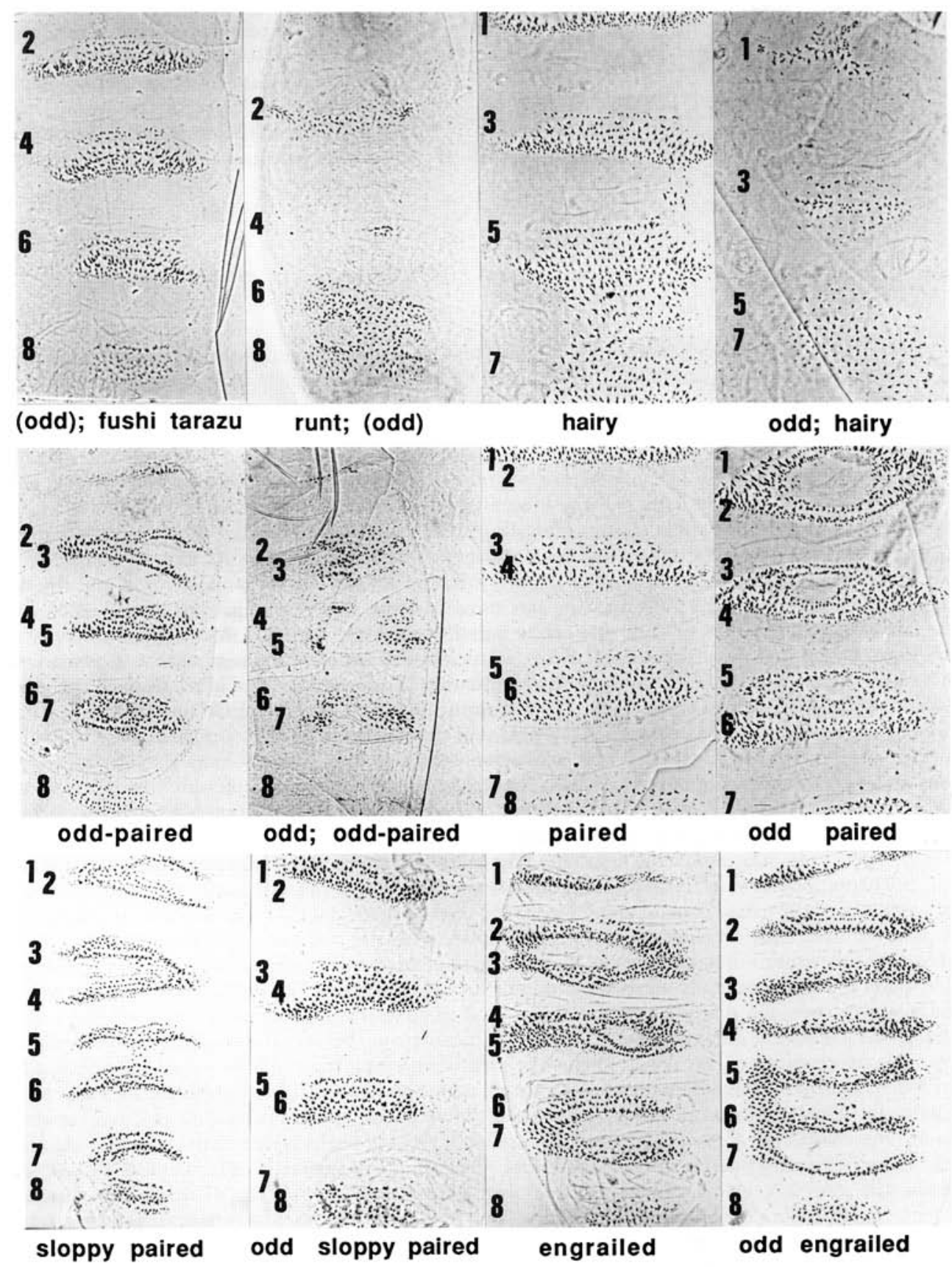

Figure 6. (See preceding page for legend.) 
The nature of pair-rule gene requirements

By analyzing the cuticle phenotypes of pair-rule double mutants, we have addressed the question of whether single mutant phenotypes indicate absolute requirements for gene activity, or whether the relationship between pattern elements and gene activity might be less direct, reflecting some form of interaction between pairrule genes. Consistent with the first alternative, several double-mutant phenotypes were essentially additive, generating only those pattern elements unaffected in either single mutant. However, other combinations readily exhibited nonadditivity, in which pattern elements eliminated by a single mutation were restored by the reduction of a second activity in the double mutant. This suggests an indirect, nonobligatory role for the pairrule genes in specifying pattern elements during embryogenesis, such that certain cell fates can derive from more than one pathway of gene activities.

The genetic interaction we have characterized most thoroughly is that between odd and eve. By examining a wide range of odd eve double-mutant combinations, we have shown that the effects of the interaction change depending on the severity of the alleles involved. Any combinations involving weak mutations in eve were essentially additive, indicating that there are thresholds of gene activity above which mutants do not interact; this threshold is not crossed in cases where the odd and eve deletion domains remain spatially separated.

Our analysis of odd eve combinations with more severe lesions revealed at least two cases where genetic interactions resulted in phenotypic suppression. The first example concerns the generation of even-numbered posterior denticle rows. As the severity of eve is increased (in combination with a given odd allele), these rows first begin to appear when the regions affected by the two mutations approach an overlap. The rows become firmly established as four partial belts when eve activity falls to a level that results in the strong pair-rule phenotype in the eve single mutant, and persist in combinations that totally lack eve activity. Because these rows alternate with the duplicated anterior rows of the odd-numbered segments, we have tentatively designated them as partial even-numbered belts. However, these denticles appear to be derived ectopically from a region anterior to that which normally gives rise to the evennumbered belts (Fig. 3d), and so do not represent the restoration of normal even-numbered rows. Because the identity of these elements is ambiguous, the polarity of the phenotypic suppression in these combinations (suppression of the odd phenotype by eve or converse) is uncertain.

The second, most dramatic example of nonadditive interactions involves the amorphic eve phenotype. When wild-type levels of odd activity are present in embryos that completely lack eve function (i.e., the eve single mutant), the ventral region of the abdomen consists of an unbroken field of denticles with no intervening naked cuticle. However, the reduction or elimination of odd activity results in the restoration of regions of naked cuticle, which serve to separate the denticles into obvious belts. Here, odd mutations clearly behave as partial suppressors of the amorphic eve phenotype, indicating that this phenotype is mediated by wild-type odd activity. Because at least some of the cells that give rise to naked cuticle in the wild-type embryo require en expression, it is interesting to note the observations of $\mathrm{Di}$ Nardo and O'Farrell (1987) concerning the patterns of en stripes in eve- mutants and odd eve double mutants. In embryos that completely lacked eve, the normal stripes of en protein (which correspond to posterior compartments of each segment) were absent; however, when both eve and odd were eliminated, some striped expression of en was restored, consistent with the reappearance of naked cuticle noted here. This indicates that the odd product somehow functions to repress $e n$ in a subset of cells in eve- embryos. ${ }^{2}$ By extension, these results further suggest that one function of eve is somehow to block this repression. Based upon the more general models described below, the eve product could either counteract the repressive effect of odd in cells where both are normally expressed (a parallel or combinatorial interaction), or it could prevent the ectopic expression of odd (hierarchical interaction).

All of the double-mutant combinations presented here involved the odd locus, raising the possibility that some special feature of this gene accounts for the cases of nonadditive interactions. Although we have sampled only a small number of the other possible combinations, certain of these have provided further examples of phenotypic suppression. For example, we have observed that a mutation in the odd-paired (opa) gene serves to suppress the amorphic eve phenotype in a fashion analogous to that described here for odd, converting the lawn phenotype into a series of discrete denticle belts separated by naked cuticle in the eve opa double mutant.

\section{The nature of pair-rule gene interactions}

We have proposed that overlapping domains of pair-rule gene expression at the blastoderm stage specify cell identities and determine the pattern elements that each cell will eventually form (Struhl 1985; Gergen et al. 1986). While it is not yet known whether all blastoderm cells become uniquely specified, molecular studies (DiNardo and O'Farrell 1987; Ingham et al. 1988) have indicated that pair-rule gene activities play an important role in establishing at least three distinct states within each segment; these correspond to cells that express en, wingless, or neither gene. Furthermore the data for en indicate that multiple pair-rule gene products are necessary to specify a domain. Although the relevant overlaps in pair-rule expression have not yet been clearly defined, the data are consistent with the combinatorial interactions envisioned in the original proposal.

In the simplest model, all pair-rule genes would func-

${ }^{2}$ While this repression is reminiscent of odd function in a wild-type background, the pattern of $e n$ staining in the double mutant represents more than the simple appearance of the ectopic domains observed in the odd phenotype (see DiNardo and O'Farrell 1987, Fig. 4). 
tion at the same level. The elimination of a given activity would alter the specification of cells in the domains where that gene is expressed, because the remaining activities would generate either nonsense or a coherent respecification to alternate positional values, depending upon the precise nature of the combinatorial code. Reducing or eliminating two different pair-rule activities could in some cases result in nonadditive effects, but only when the two domains of expression overlap and only in those cells that normally express both genes.

The model discussed above assumes that the only interactions among pair-rule genes occur in parallel. In fact, several studies have demonstrated that the precise spatial patterning of a variety of pair-rule genes is altered by mutations in other members of the class (e.g., Carroll and Scott 1986; Harding et al. 1986; Howard and Ingham 1986). Consequently, any two pair-rule genes could potentially interact in a cross-regulatory or hierarchical sequence. Such regulatory interactions could be responsible for the phenotypic suppression observed in double mutants, at least in cases where one gene normally functions to restrict the spatial domain of a second, downstream gene. While mutations in the first gene would cause ectopic expression of the second, the effects of this expression would be mitigated in the double $\mathrm{mu}$ tant (see, for example, the discussion of the odd en phenotype presented above).

In the absense of molecular data it is not possible to distinguish between parallel (combinatorial) and hierarchical levels of interaction. However, the two models are not mutually exclusive, and a given pair-rule gene might function at both levels. Regulatory interactions among pair-rule genes would be essential to refine and/ or stabilize the overlapping domains of expression, but once a stable pattern is established, all gene products could act in parallel. In this case, segmentation phenotypes would depend on the net effect of disrupting both functions, at least in amorphic mutants; if the two functions required different thresholds of activity, the analysis of hypomorphic alleles might allow the two roles to be distinguished.

Indeed, it is possible that the two examples of nonadditivity we have noted in odd eve double mutants reflect these two levels of interaction. For example, the posterior rows of denticles that appear between the odd-numbered denticle rows in various odd eve combinations exhibit two features predicted by the simple combinatorial model: These elements are only observed when the two pattern deletions approach or exceed an overlap, and appear to derive ectopically from regions of naked cuticle where this overlap occurs. This suggests that a subset of blastoderm cells that have moderate requirements for both odd and eve activity give rise to these denticles in the double mutants. These cells would die or secrete naked cuticle when either activity alone was reduced below the required threshold, but would be respecified to generate the observed denticle rows upon the reduction of both activities.

Meanwhile, the amorphic eve phenotype and the restoration of naked cuticle upon reduction of odd activity would readily be explained by assuming a hierarchical interaction. One function of eve might be to limit the spatial domain of odd expression. Complete absence of eve function would generate a lawn of odd-numbered denticles due to broadening of the odd domain. The reduction of odd activity in the double mutant would eliminate the effects of this ectopic expression, shifting cell fates from denticle elements back to naked cuticle. The observation that weak eve mutations are not affected by loss of odd function argues that the weak eve phenotype does not result from ectopic odd expression. Moreover, since eve mutations do not significantly alter the odd pattern deletions, it is unlikely that the odd product functions to repress eve expression (see also Frasch and Levine 1987).

Finally, it is possible that novel pattern elements in double mutants result from indirect interactions between pair-rule genes which are expressed in different cells. Both mechanisms proposed above assume that segmentation phenotypes primarily reflect autonomous requirements for gene activity (Gergen and Wieschaus 1985), and that most pair-rule genes interact within particular cells to determine developmental fates. It is well established that at least one function of the pair-rule genes is the proper spatial activation of stably expressed genes in the segment polarity and homeotic classes (see Akam 1987 and references therein). It is likely that this initial regulation occurs in a cell autonomous fashion. However, cell interactions are required for the maintenance and/or further elaboration of the segmental pattern (DiNardo et al. 1988; Martinez-Arias et al. 1988), and nonautonomous effects have been described for at least one of these genes (wingless, Morata and Lawrence 1977; Baker 1988; see also Wieschaus and Riggleman 1987). This indicates that altered patterns of cell communication should result as a secondary consequence of pair-rule mutations. Where such mutations result in significant changes in the expression of segment polarity genes, the final pattern may include a substantial nonautonomous component.

\section{Materials and methods \\ Genotypes and stocks}

Unless otherwise indicated, mutations were derived from screens designed to isolate ethylmethane sulfonate (EMS)-induced, recessive zygotic lethals which alter the segmental pattern (Jürgens et al. 1984; Nüsslein-Volhard et al. 1984; Wieschaus et al. 1984), and maintained as balanced stocks. The phenotypes of seven odd mutants were examined, including four EMS-induced alleles $(I I I D, I I I C, 7 L$, and $9 P)$, two obtained in hybrid dysgenic screens (hd1 and $h d 2$, derived from stocks obtained from M. Simmons), and one X-ray-induced allele (1.36; Nüsslein-Volhard et al. 1985). Although no discrete, cytologically visible chromosomal deficiency for the odd locus was available, the phenotype associated with strong alleles is similar to that observed in aneuploid crosses designed to generate a synthetic deficiency for the locus (Nüsslein-Volhard et al. 1985).

Double-mutant chromosomes bearing odd and other second chromosomal loci were generated by meiotic recombination and maintained as balanced stocks over CyO. These included all nine odd eve combinations involving odd IIID, IIIC, and $7 \mathrm{~L}$ and eve IIR, ID, and $D f(2 R) e v e^{1.27}$ (Nüsslein-Volhard et al. 
1985); the six combinations between these same odd alleles and prd $I I N$ and $I I B$; the four combinations between odd $I I I C$ and $7 L$ and sIp IIM and $7 L$; and combinations of oddIIID with both $e n^{I O}$ and $e n^{I K}$. Phenotypic descriptions are based on the examination of cuticle preparations (Wieschaus and NüssleinVolhard 1986) from 30-100 mutant embryos per stock. To generate double mutants between odd and X-linked (runt) or third-chromosomal (ftz, hairy, opa) loci, flies from each balanced stock were mated, and unbalanced $F_{1}$ heterozygotes bearing both mutant alleles were identified by the absence of both balancer chromosomes. Siblings were mated to generate mutant embryos that were homozygous for either single locus alone, or were doubly mutant at an expected ratio of $3: 3: 1$. At least 80 mutant embryos from each cross were examined; embryos that resembled either single mutant were counted as separate pools and compared to those with ambiguous or novel phenotypes. With opa and hairy, the expected $3: 3: 1$ ratios were observed among the unhatched embryos, and the latter class was assumed to represent double mutants; with runt and fushi tarazu (ftz), virtually all embryos were scored as either single mutant in a $4: 3$ ratio (runt: odd and ftz: odd, respectively).

\section{Acknowledgments}

We thank Christiane Nüsslein-Volhard for providing stocks, Dari Sweeton for expert darkroom assistance, and Trudi Schupbach and numerous members of the lab for advice, encouragement, discussions, and critical readings of the manuscript. These experiments were supported by National Institutes of Health grant 5RO1HD22780 to E.W. and an NIH postdoctoral fellowship to D.C.

\section{References}

Akam, M. 1987. The molecular basis for metameric pattern in the Drosophila embryo. Development 101: 1-22.

Baker, N.E. 1988. Embryonic and imaginal requirements for wingless, a segment polarity gene in Drosophila. Dev. Biol. 125: $96-108$.

Carroll, S.B. and M.P. Scott. 1986. Zygotically active genes that affect the spatial expression of the fushi tarazu segmentation gene during early Drosophila embryogenesis. Cell 45: $113-126$.

DiNardo, S. and P.H. O'Farrell. 1987. Establishment and refinement of segmental pattern in the Drosophila embryo: spatial control of engrailed expression by pair-rule genes. Genes Dev. 1: 1212-1225.

DiNardo, S., E. Sher, J. Heemskerk-Jongens, J.A. Kassis, and P.H. O'Farrell. 1988. Two-tiered regulation of spatially patterned engrailed gene expression during Drosophila embryogenesis. Nature 332: 604-609.

Frasch, M. and M. Levine. 1987. Complementary patterns of even-skipped and fushi tarazu expression involve their differential regulation by a common set of segmentation genes in Drosophila. Genes Dev. 1: 981-995.

French, V., P.J. Bryant, and S.V. Bryant. 1976. Pattern regeneration in epimorphic fields. Science 193: 969-981.

Gergen, J.P., D. Coulter, and E.F. Wieschaus. 1986. Segmental pattern and blastoderm cell identities. Symp. Soc. Dev. Biol. 43: $195-220$.

Gergen, J.P. and E.F. Wieschaus. 1985. The localized requirements for a gene affecting segmentation in Drosophila: Analysis of larvae mosaic for runt. Dev. Biol. 109: 321-335.

- 1986. Dosage requirements for runt in the segmentation of Drosophila embryos. Cell 45: 289-299.

Harding, K., C. Rushlow, H.J. Doyle, T. Hoey, and M. Levine. 1986. Cross-regulatory interactions among pair-rule genes in
Drosophila. Science. 233: 953-959.

Howard, K. and P. Ingham. 1986. Regulatory interactions between the segmentation genes fushi tarazu, hairy, and en grailed in the Drosophila blastoderm. Cell 44: 949-957.

Ingham, P.W., N.E. Baker, and A. Martinez-Arias. 1988. Regulation of segment polarity genes in the Drosophila blastoderm by fushi-tarazu and even-skipped. Nature 331: 73-75.

Ingham, P.W., S.M. Pinchin, K.R. Howard, and D. Ish-Horowicz. 1985. Genetic analysis of the hairy locus in Drosophila melanogaster. Genetics 111: 463-486.

Jürgens, G. 1987. Segmental organization of the tail region in the embryo of Drosophila melanogaster. Wilheim Roux's Arch. Dev. Biol. 196: 141-157.

Jürgens, G., R. Lehmann, M. Schardin, and C. Nüsslein-Volhard. 1986. Segmental organization of the head in the embryo of Drosophila melanogaster. Wilhelm Roux's Arch. Dev. Biol. 195: 359-377.

Jürgens, G., E. Wieschaus, C. Nüsslein-Volhard, and $H$. Kluding. 1984. Mutations affecting the pattern of the larval cuticle in Drosophila melanogaster. II Zygotic loci on the third chromosome. Wilhelm Roux's Arch. Dev. Biol. 193: 283-295.

Kornberg, T., I. Siden, P. O'Farrell, and M. Simon. 1985. The engrailed locus of Drosophila: In situ locialization of transcripts reveals compartment-specific expression. Cell 40: $45-53$.

MacDonald, P.M., P. Ingham, and G. Struhl. 1986. Isolation, structure, and expression of even-skipped: A second pairrule gene of Drosophila containing a homeo box. Cell 47: $721-734$.

Martinez-Arias, A. and P.A. Lawrence. 1985. Parasegments and compartments in the Drosophila embryo. Nature 313: 639642.

Martinez-Arias, A., N.E. Baker, and P.W. Ingham. 1988. Role of segment polarity genes in the definition and maintenance of cell states in the Drosophila embryo. Development 103: $157-170$.

Morata, G. and P.A. Lawrence. 1977. The development of wingless, a homeotic mutation of Drosophila melanogaster. Dev. Biol. 56: 227-240.

Nüsslein-Volhard, C. and E. Wieschaus. 1980. Mutations affecting segment number and polarity in Drosophila. Nature 287: 795-801.

Nüsslein-Volhard, C., E. Wieschaus, and H. Kluding. 1984. Mutations affecting the pattern of the larval cuticle in Drosophila melanogaster. I. Zygotic loci on the second chromosome. Wilhelm Roux's Arch. Dev. Biol. 193: 267-282.

Nüsslein-Volhard, C., H. Kluding, and G. Jürgens. 1985. Genes affecting the segmental subdivision of the Drosophila embryo. Cold Spring Harbor Symp. Quant. Biol. 50: 145-154.

O'Farrell, P. and M. Scott. 1986. Spatial programming of gene expression in early Drosophila embryogenesis. Annu. Rev. Cell Biol. 2: 49-80.

Struhl, G. 1985. Near-reciprocal phenotypes caused by inactivation or indiscriminate expression of the Drosophila segmentation gene fushi tarazu. Nature 318: 677-680.

Wieschaus, E., C. Nüsslein-Volhard, and G. Jürgens. 1984. Mutations affecting the pattern of the larval cuticle in Drosophila melanogaster II. Zygotic loci on the $\mathrm{X}$ chromosome and the fourth chromosome. Wilhelm Roux's Arch. Dev. Biol. 193: 296-307.

Wieschaus, E. and C. Nüsslein-Volhard. 1986. Looking at embryos. In Drosophila: A practical approach. (ed. D.B. Roberts| pp.199-228. IRL Press Ltd., Oxford, England.

Wieschaus, E. and R. Riggleman. 1987. Autonomous requirements for the segment polarity gene armadillo during Drosophila embryogenesis. Cell 49: 177-184. 


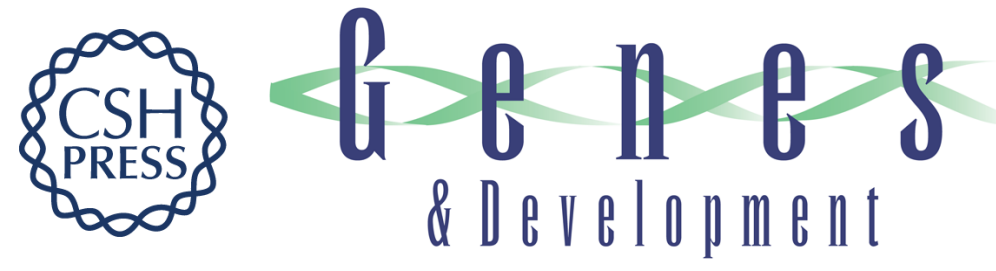

\section{Gene activities and segmental patterning in Drosophila: analysis of odd-skipped and pair-rule double mutants.}

D E Coulter and E Wieschaus

Genes Dev. 1988, 2:

Access the most recent version at doi:10.1101/gad.2.12b.1812

References This article cites 29 articles, 7 of which can be accessed free at:

http://genesdev.cshlp.org/content/2/12b/1812.full.html\#ref-list-1

License

Email Alerting

Service

Receive free email alerts when new articles cite this article - sign up in the box at the top right corner of the article or click here.

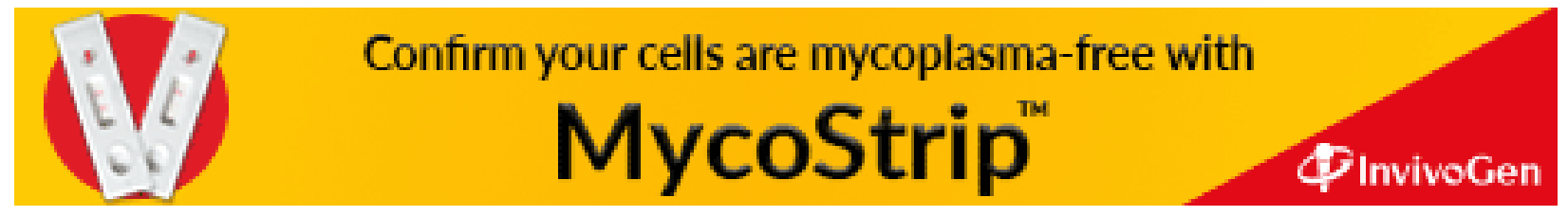

